\title{
Integral Geometry and Real Zeros of Thue-Morse Polynomials
}

\author{
Christophe Doche and Michel Mendès France
}

Dedicated to the memory of Gian-Carlo Rota

\section{CONTENTS}

1. Finite curves

2. Breadth of a Bounded Set

3. Extension to Finite Doodles

4. Dimension

5. Real Zeros of Real Polynomials

6. Deterministic Results

7. Elementary Properties

8. Thue-Morse Polynomials of Odd Degree

9. Thue-Morse Polynomials of Even Degree

10. Proof of the Theorems

Acknowledgements

Addendum

References

Keywords: Integral geometry, Thue-Morse sequence, real roots
We study the average number of intersecting points of a given curve with random hyperplanes in an n-dimensional Euclidean space. As noticed by A. Edelman and E. Kostlan, this problem is closely linked to finding the average number of real zeros of random polynomials. They showed that a real polynomial of degree $\mathrm{n}$ has on average $\frac{2}{\pi} \log \mathrm{n}+\mathrm{O}(1)$ real zeros (M. Kac's theorem).

This result leads us to the following problem: given a real sequence $\left(\alpha_{\mathrm{k}}\right)_{\mathrm{k} \in \mathbb{N}}$, study the average

$$
\frac{1}{N} \sum_{n=0}^{N-1} \rho\left(f_{n}\right),
$$

where $\rho\left(\mathrm{f}_{\mathrm{n}}\right)$ is the number of real zeros of $\mathrm{f}_{\mathrm{n}}(\mathrm{X})=\alpha_{0}+\alpha_{1} \mathrm{X}+\cdots+$ $\alpha_{\mathrm{n}} \mathrm{X}^{\mathrm{n}}$. We give theoretical results for the Thue-Morse polynomials and numerical evidence for other polynomials.

\section{FINITE CURVES}

Let $\mathcal{E}_{n}$ be real $n$-dimensional Euclidean affine space with a given orthonormal base. We identify points $x \in \mathcal{E}_{n}$ with the column vector ${ }^{t}\left(x_{1}, \ldots, x_{n}\right)$ whose entries are the coordinates of $x$.

A hyperplane $h \subset \mathcal{E}_{n}$ which does not contain the origin is represented by its Cartesian equation

$$
\sum_{i=1}^{n} h_{i} x_{i}=1,
$$

or $h x=1$ for short, where $h=\left(h_{1}, h_{2}, \ldots, h_{n}\right)$ is an element of the dual space $\mathcal{E}_{n}^{*}$. Both $\mathcal{E}_{n}$ and $\mathcal{E}_{n}^{*}$ are endowed with the Euclidean norm.

A change of orthonormal base in $\mathcal{E}_{n}$ induces a change of coordinates from the initial base to the new one:

$$
x \mapsto x^{\prime}=A x+x^{0}
$$


where $A$ is an $n \times n$ orthogonal matrix. The hyperplane $h$ then becomes $h^{\prime}=h A^{-1} /\left(1+h A^{-1} x^{0}\right)$ and it is easy to see that

$$
\left\|h^{\prime}\right\|^{-n-1} \prod_{i=1}^{n} d h_{i}^{\prime}=\|h\|^{-n-1} \prod_{i=1}^{n} d h_{i},
$$

so that

$$
d h=\|h\|^{-n-1} \prod_{i=1}^{n} d h_{i}
$$

is the natural invariant measure on the space $\mathcal{E}_{n}^{*}$. Perhaps the most elementary way to see this classical result is to notice that any change of orthogonal base is a product of a rotation around the origin followed by $n$ translations parallel to a coordinate axis. For each of these component base changes, the above invariance holds.

Let $\gamma \subset \mathcal{E}_{n}$ be a finite rectifiable curve, that is, a rectifiable curve of finite length $|\gamma|$. Let $\Omega(\gamma)$ be the family of hyperplanes $h$ that intersect $\gamma$, and let $|\gamma \cap h|$ be the number of intersection points of $h$ and $\gamma$. Santaló [1976, p. 245] establishes that

$$
\int|\gamma \cap h| d h=\frac{\pi^{(n-1) / 2}}{\Gamma\left(\frac{n+1}{2}\right)}|\gamma|,
$$

where $\Gamma$ is the usual Euler gamma function. See also [Klain and Rota 1997].

\section{BREADTH OF A BOUNDED SET}

Let $K$ be a bounded convex set in $\mathcal{E}_{n}$. For $x \in \mathcal{E}_{n}$ call $K_{x}$ the length of the orthogonal projection of $K$ on the axis $O x$, where $O$ is the origin. Lengths are positive. The mean breadth of $K$ is

$$
\int_{\mathbb{S}_{n}} K_{x} d x / \int_{\mathbb{S}_{n}} d x
$$

where $\mathbb{S}_{n}$ is the unit sphere $x_{1}^{2}+x_{2}^{2}+\cdots+x_{n}^{2}=1$.

If $L$ is any bounded set in $\mathcal{E}_{n}$, we define the mean breadth $B(L)$ as the mean breadth of the convex hull of $L$; in particular, the mean breadth of $\gamma$ is well defined. Using Santaló's result again, we easily find that the $h$-measure of those hyperplanes $h$ that intersect $\gamma$ is

$$
\int_{h \cap \gamma \neq \varnothing} d h=\frac{\pi^{n / 2}}{\Gamma\left(\frac{n}{2}\right)} B(\gamma) .
$$

The quotient of equalities (1-1) and (2-1) thus gives us the average number $N(\gamma)$ of intersecting points of $\gamma$ with a random hyperplane given that these hyperplanes meet $\gamma$ :

$$
N(\gamma)=\frac{1}{\sqrt{\pi}} \frac{\Gamma\left(\frac{n}{2}\right)}{\Gamma\left(\frac{n+1}{2}\right)} \frac{|\gamma|}{B(\gamma)} .
$$

Let $\delta$ be the diameter of the set $\gamma$. Then clearly $B(\gamma) \leqslant \delta$. On the other hand the convex hull of $\gamma$ contains a segment $\sigma$ of length $\delta$. Since

$$
B(\gamma) \geqslant B(\sigma)=\frac{1}{\sqrt{\pi}} \frac{\Gamma\left(\frac{n}{2}\right)}{\Gamma\left(\frac{n+1}{2}\right)} \delta,
$$

where the equality is easily verified, we conclude that

$$
\frac{1}{\sqrt{\pi}} \frac{\Gamma\left(\frac{n}{2}\right)}{\Gamma\left(\frac{n+1}{2}\right)} \frac{|\gamma|}{\delta} \leqslant N(\gamma) \leqslant \frac{|\gamma|}{\delta} .
$$

\section{EXTENSION TO FINITE DOODLES}

Formulas (2-2) and (2-3) can be extended to generalized curves called "doodles". A doodle $\gamma$ is a connected set which is a finite union of rectifiable curves $\gamma_{1}, \gamma_{2}, \ldots, \gamma_{k}$. The length of $\gamma$ is naturally defined as

$$
|\gamma|=\sum_{i=1}^{k}\left|\gamma_{i}\right|
$$

To any doodle $\gamma$ we can associate a closed curve $\tilde{\gamma}$ that goes over $\gamma$ twice. According to formula (2-2),

$$
N(\tilde{\gamma})=\frac{1}{\sqrt{\pi}} \frac{\Gamma\left(\frac{n}{2}\right)}{\Gamma\left(\frac{n+1}{2}\right)} \frac{2|\gamma|}{B(\gamma)},
$$

since $|\tilde{\gamma}|=2|\gamma|$ and $B(\tilde{\gamma})=B(\gamma)$. But $N(\tilde{\gamma})=$ $2 N(\gamma)$ since each intersection point of a hyperplane $h$ with $\tilde{\gamma}$ counts twice. Dividing by 2 leads to formula (2-2) for doodles.

This result should be compared to those of Favard [1932], Sulanke [1966] and Laurent-Gengoux (private communication).

\section{DIMENSION}

Several earlier articles have discussed the dimension of curves in the plane [Mendès France and Tenenbaum 1981; Dekking and Mendès France 1981; Mendès France 1991]. The extension to curves in $\mathcal{E}_{n}$ is straightforward.

Indeed, let $\gamma$ be an unbounded, locally rectifiable curve in $\varepsilon_{n}$ such that bounded subsets of $\varepsilon_{n}$ contain only finite portions of $\gamma$. So for example in $\varepsilon_{2}$ curves 
like the one given in polar coordinates by $\theta=\sin 1 / \rho$ are excluded: the unit ball centered at the origin contains infinitely many branches of the curve.

With no real loss of generality we assume that $\gamma$ has an endpoint (or rather a starting point!). Let $\gamma_{s}$ denote the beginning portion of $\gamma$ of length $s$. Let $\varepsilon>0$ be given. Consider the so-called Minkowski $\varepsilon$-sausage

$$
\gamma(s, \varepsilon)=\left\{x \in \mathcal{E}_{n} \mid \operatorname{dist}\left(x, \gamma_{s}\right) \leqslant \varepsilon\right\} .
$$

Let $|\gamma(s, \varepsilon)|$ be its volume. If $\delta(s)$ is the diameter of $\gamma_{s}$, define the dimension $d=\operatorname{dim}(\gamma)$ by the formula

$$
\operatorname{dim}(\gamma)=\liminf _{s \rightarrow \infty} \frac{\log |\gamma(s, \varepsilon)|}{\log \delta(s)} .
$$

Despite appearances, $\operatorname{dim}(\gamma)$ does not depend on $\varepsilon$ : this is easily seen [Mendès France 1991, page 329]. Clearly $1 \leqslant d \leqslant n$, and it can be shown that for all $\alpha \in[1, n]$ there exists a curve $\gamma$ for which $\operatorname{dim}(\gamma)=$ $\alpha$.

The volume of $\gamma(s, \varepsilon)$ is at most

$$
|\gamma(s, \varepsilon)| \leqslant \frac{\pi^{(n-1) / 2}}{\Gamma\left(\frac{n+1}{2}\right)} \varepsilon^{n-1} s+O\left(\varepsilon^{n}\right),
$$

so that

$$
\operatorname{dim}(\gamma) \leqslant \liminf _{s \rightarrow \infty} \frac{\log s}{\log \delta(s)} .
$$

Under certain circumstances equality holds: for example, when there exists a real number $A>0$ such that unit balls contain portions of $\gamma$ of total length less than $A$.

Let $\varepsilon>0$ be given. Formula (4-2) shows that there exists a value $s(\varepsilon)$ such that

$$
\frac{\log \delta(s)}{\log s} \leqslant \frac{1}{d}+\frac{\varepsilon}{2}
$$

for all $s>s(\varepsilon)$, so that

$$
\delta(s) \leqslant s^{1 / d+\varepsilon / 2} .
$$

Inequality $(2-3)$ therefore implies a lower bound for $N\left(\gamma_{s}\right)=: N(s)$ :

$$
N(s) \geqslant \frac{1}{\sqrt{\pi}} \frac{\Gamma\left(\frac{n}{2}\right)}{\Gamma\left(\frac{n+1}{2}\right)} s^{1-1 / d-\varepsilon / 2} \geqslant s^{1-1 / d-\varepsilon} .
$$

On the other hand, if $\gamma$ is a curve for which formula $(4-2)$ is an equality, then

$$
N(s) \leqslant s^{1-1 / d+\varepsilon}
$$

for infinitely many large $s$.
Inequality (4-3) implies that if $d>1$ the average number of intersections of $\gamma_{s}$ with a random hyperplane tends to infinity as $s$ grows. In particular, if $\gamma$ is an unbounded algebraic curve then $N(s)$ is less than the degree of $\gamma$ : this forces $d=1$. Unbounded algebraic curves are one-dimensional.

\section{REAL ZEROS OF REAL POLYNOMIALS}

Let $\gamma$ be an unbounded curve in $\mathcal{E}_{n}$ as before. Let

$$
x_{1}=\varphi_{1}(s), \quad x_{2}=\varphi_{2}(s), \quad \ldots, \quad x_{n}=\varphi_{n}(s)
$$

be its parametrical representation: the $\varphi_{i}$ 's are real functions of bounded variation on every finite interval and $s$ denotes the length coordinate. The previous results show that the average number of real zeros of the equation

$$
\sum_{i=1}^{n} h_{i} \varphi_{i}(t)-1=0, \quad \text { for } 0 \leqslant t \leqslant s
$$

is

$$
N(s) \geqslant s^{1-1 / d-\varepsilon}
$$

for all large $s$.

The most interesting case is when $\varphi_{i}(t)=t^{i}$, with $|t| \leqslant T \sim s^{1 / n}$, for $i=1,2, \ldots, n$. Then $N(s)$ would be the average number of real zeros in $[-T,+T]$ of the polynomial

$$
P(t) \equiv h_{n} t^{n}+h_{n-1} t^{n-1}+\cdots+h_{1} t-1 .
$$

Unfortunately, as noticed in Section 4, the dimension is 1 so all we get is the trivial result $N(s)>0$.

Edelman and Kostlan [1995], reflecting on a result of M. Kac $[1943 ; 1949 ; 1959]$, realized that it is possible - and even easy - to obtain the average number of real zeros of the polynomial

$$
h_{0}+h_{1} t+\cdots+h_{n} t^{n}
$$

using results from integral geometry. Indeed, consider the curve

$$
\left(x_{0}, \ldots, x_{n}\right)=\left(1, t, t^{2}, \ldots, t^{n}\right), \quad \text { for } t \in \mathbb{R},
$$

or rather the curve $\gamma$ obtained by projecting it centrally onto the unit sphere $x_{0}^{2}+\cdots+x_{n}^{2}=1$. Intersecting $\gamma$ by a random hyperplane $h$

$$
h_{0} x_{0}+h_{1} x_{1}+\cdots+h_{n} x_{n}=0,
$$

where $h=\left(h_{0}, h_{1}, \ldots, h_{n}\right)$ is uniformly distributed on the surface of the unit sphere $h_{0}^{2}+\cdots+h_{n}^{2}=$ 1 , shows that the average number of intersecting 
points - that is, zeros of the polynomial $h_{0}+h_{1} t+$ $\cdots+h_{n} t^{n}$ - is equal to $|\gamma| / \pi$. The length $|\gamma|$ is easily computed:

$$
\frac{1}{\pi}|\gamma|=\frac{1}{\pi} \int_{-\infty}^{+\infty} \sqrt{\frac{1}{\left(t^{2}-1\right)^{2}}-\frac{(n+1)^{2} t^{2 n}}{\left(t^{2 n+2}-1\right)^{2}}} d t .
$$

This is Kac's formula, obtained by Edelman and Kostlan's analysis. Kac obtained an equivalence when $n$ increases to infinity and the second authors were able to give a much more precise result which finally shows that the average number of real zeros of the polynomial is

$$
\frac{2}{\pi} \log n+0.625 \ldots+\frac{2}{\pi n}+O\left(\frac{1}{n^{2}}\right) .
$$

The main difference between their approach and ours holds in projecting curves on the surface of the unit $n$-sphere so that curves of infinite length in $\mathcal{E}_{n+1}$ may well have a finite length on $\mathbb{S}_{n}$. Their normalizing factor $1 / \pi$ is a measure of the set of hyperplanes $h \in \mathcal{E}^{n+1}$ which pass through the origin. Some of the hyperplanes do not intersect $\gamma$.

Erdős and Offord [1956] discussed a similar problem, namely to compute the average number of real zeros of a $n$ degree random polynomial with coefficients \pm 1 . They find that the average is again $\frac{2}{\pi} \log n$. The method is very different and it would be desirable to be able to find a geometrical approach to their analysis.

\section{DETERMINISTIC RESULTS}

The deterministic counterpart of the Kac-ErdősOfford theorems could be as follows. Given an infinite sequence $\alpha=\left(\alpha_{n}\right)_{n \in \mathbb{N}}$ of \pm 1 , say the ErdösOfford case, we form the polynomials

$$
f_{n}(X)=\alpha_{0}+\alpha_{1} X+\cdots+\alpha_{n} X^{n} .
$$

Writing $\rho\left(f_{n}\right)$ for the number of real zeros of $f_{n}$ counted with multiplicity, we want to study the average

$$
\frac{1}{N} \sum_{n=0}^{N-1} \rho\left(f_{n}\right)
$$

and to compare it with $\frac{2}{\pi} \log N$ as $N$ goes to infinity. If the two quantities are equivalent we can consider the sequence $\left(\alpha_{n}\right)_{n \in \mathbb{N}}$ to be "random" in some sense. We conjecture that almost all sequences $\alpha \in\{-1,1\}^{\mathbb{N}}$ behave like that. We studied two deterministic $(+,-)$ sequences. The first is the ThueMorse sequence, which is the only nontrivial example for which we can give a precise result. It is also a special case of more interesting sequences (see addendum at the end of this paper for details). Even though the Thue-Morse sequence is far from mimicking randomness, it still retains some features associated with randomness: namely, the spectral measure is continuous (yet not absolutely continuous as would be the case for a random sequence). Theorems 6.1 and 6.2 below show that the ThueMorse sequence is actually far from random.

Before stating them, we consider another $(+,-)$ sequence which appears to behave à la Kac-ErdősOfford. Put $\beta_{0}=\beta_{1}=1$ and $\beta_{n} \equiv p_{n} \bmod 4$ for $n \geqslant 2$, where $p_{n}$ is the $n$-th prime number. To this day it seems completely out of reach to prove any relevant theorem concerning the zeros of the related polynomials. So we leave it as a conjecture to establish that the average is equivalent to $\frac{2}{\pi} \log N$. See our numerical evidence obtained with the help of the command polsturm of PARI-GP up to $N=600$. Figure 1 shows

$$
\frac{\pi}{2 N \log N} \sum_{n=0}^{N-1} \rho\left(f_{n}\right)
$$

as a function of $N$ when the coefficients of $f_{n}$ follow a random sequence and the $\beta$ sequence mentioned above.

In the remainder of the paper, $\left(\varepsilon_{i}\right)_{i \in \mathbb{N}}$ will represent the Thue-Morse sequence defined by $\varepsilon_{i}=$ $(-1)^{\nu(i)}$ where $\nu(i)$ is the sum of the binary digits of $i$. Then $f_{n}(X)$ will be the Thue-Morse polynomial of degree $n$, that is, $f_{n}(X)=\varepsilon_{0}+\varepsilon_{1} X+\cdots+\varepsilon_{n} X^{n}$. For this very special sequence we are able to prove that

$$
\lim _{N \rightarrow \infty} \frac{1}{N \log N} \sum_{n=0}^{N-1} \rho\left(f_{n}\right)=0 .
$$

In fact, we shall show:

Theorem 6.1. If $n \in \mathbb{N}$ is even, $f_{n}$ has at most two real roots. More precisely:

1. If $n \equiv 0 \bmod 4$ and $\varepsilon_{n}=1$ then $f_{n}$ has no real root.

2. If $n \equiv 2 \bmod 4$ and $\varepsilon_{n}=1$ then $f_{n}$ has two negative roots. 

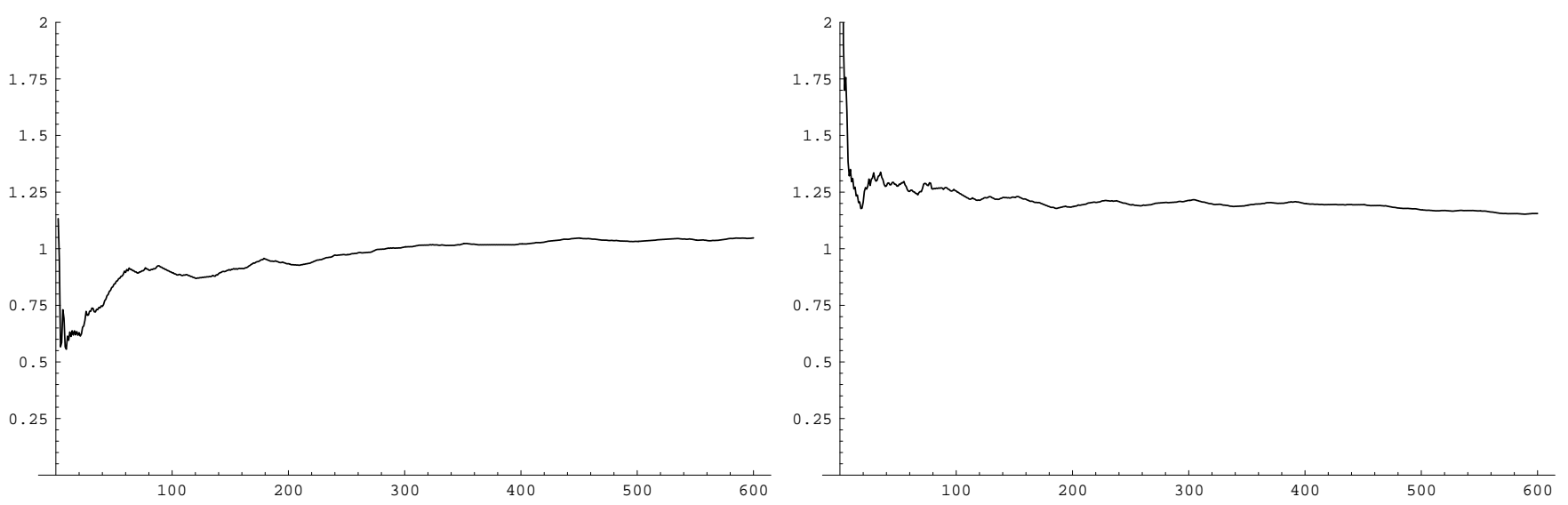

FIGURE 1. Plot of $\pi /(2 N \log N) \sum_{n=0}^{N-1} \rho\left(f_{n}\right)$ as a function of $N$, for a random sequence (left) and the $\beta$ sequence of the text (right).

3. If $\varepsilon_{n}=-1$ then $f_{n}$ has two real roots, one positive and the other negative.

4. If $n \in \mathbb{N}$ is odd, let $k$ be the 2-adic valuation of $n+1$. Then if $\varepsilon_{n}=(-1)^{k}, f_{n}$ has $2 k-1$ real roots and if $\varepsilon_{n}=(-1)^{k+1}, f_{n}$ has $2 k+1$ real roots.

Theorem 6.2. The mean

$$
\frac{1}{N} \sum_{n=0}^{N-1} \rho\left(f_{n}\right)
$$

tends to $\frac{11}{4}$ as $N$ tends to infinity.

We start with elementary results.

\section{ELEMENTARY PROPERTIES}

First we remark that $\varepsilon_{2 i}=\varepsilon_{i}$ and $\varepsilon_{2 i+1}=-\varepsilon_{2 i}$ and that the generating series of $\left(\varepsilon_{i}\right)_{i \in \mathbb{N}}$ is

$$
\sum_{i=0}^{\infty} \varepsilon_{i} X^{i}=\prod_{l=0}^{\infty}\left(1-X^{2^{l}}\right) .
$$

Now we define the Thue-Morse word $\mathcal{E}$ on the alphabet $\{+,-\}$ by iterating the morphism $\varphi$ defined by $\varphi(+)=+-$ and $\varphi(-)=-+$ so that

$$
\mathcal{E}=\lim _{n \rightarrow \infty} \varphi^{n}(+) .
$$

The link between the Thue-Morse word and the Thue-Morse sequence is well known. The letter of order $i$ of $\mathcal{E}$ is + or - depending on whether $\varepsilon_{i}=1$ or $\varepsilon_{i}=-1$. We introduce another useful morphism, namely $\psi$ satisfying $\psi(+)=+--+$ and $\psi(-)=-++-$. Since $\psi=\varphi^{2}$, we have also

$$
\mathcal{E}=\lim _{n \rightarrow \infty} \psi^{n}(+) \text {. }
$$

At last, if $i \leqslant j$ we write ${ }_{i} \mathcal{E}_{j}$ for the factor of $\mathcal{E}$ whose first (resp. last) letter is the letter of rank $i$ (resp. $j$ ) of $\mathcal{E}$. For example ${ }_{0} \mathcal{E}_{3}=+--+$. In the same way, the definitions of $\psi$ and $\varphi$ ensure that

$$
{ }_{4 n} \mathcal{E}_{4 n+3}=\psi(+) \text { or } \psi(-) .
$$

and that +++ and --- are never factors of $\mathcal{E}$.

We now get back to polynomials. We write $\mathcal{R}(P)$ for the set of real roots of a polynomial $P$. Note that in general $|\mathcal{R}(P)| \neq \rho(P)$ since $\rho(P)$ counts the roots with multiplicity. If we consider a polynomial $P$ with coefficients \pm 1 it is well known that $\mathcal{R}(P) \subset\left[-2,-\frac{1}{2}\right] \cup\left[\frac{1}{2}, 2\right]$. In addition, for ThueMorse polynomials, we have:

Lemma 7.1. $\mathcal{R}\left(f_{n}\right) \cap[-0.95,0.95]=\varnothing$ for $n>255$.

Proof. Indeed,

$$
\left|f_{n}(x)-f_{255}(x)\right| \leqslant 0.95^{256} \frac{1}{1-0.95} .
$$

Now $(7-1)$ shows that $f_{255}(x)=\prod_{l=0}^{7}\left(1-x^{2^{l}}\right)$ so that for $x \in[-0.95,0.95]$ we have

$$
f_{255}(x) \geqslant f_{255}(0.95)=0.000132 \ldots,
$$

whereas $0.95^{256} \frac{1}{1-0.95} \leqslant 0.00004$.

We now investigate odd degrees.

\section{THUE-MORSE POLYNOMIALS OF ODD DEGREE}

Lemma 8.1. If $n \equiv-1 \bmod 2^{k}$, namely if $n=v 2^{k}+$ $2^{k}-1$ with $v \geqslant 0$, then

$$
f_{n}(X)=f_{2^{k}-1}(X) f_{v}\left(X^{2^{k}}\right) .
$$


Proof. We remark that every integer $i \in[0, n]$ can be written uniquely as the sum $i=2^{k} q+r$ with $0 \leqslant$ $q \leqslant v$ and $0 \leqslant r \leqslant 2^{k}-1$. Since $\varepsilon_{i}=\varepsilon_{r} \varepsilon_{q}$, we have

$$
\begin{aligned}
f_{n}(X) & =\sum_{i=0}^{n} \varepsilon_{i} X^{i}=\sum_{r=0}^{2^{k}-1} \sum_{q=0}^{v} \varepsilon_{r} X^{r} \varepsilon_{q} X^{q 2^{k}} \\
& =f_{2^{k}-1}(X) f_{v}\left(X^{2^{k}}\right) .
\end{aligned}
$$

Now we specify the roots of $f_{2^{k}-1}$.

Lemma 8.2. Let $k$ be a positive integer. The only real roots of $f_{2^{k}-1}$ are -1 and 1 respectively of order $k-1$ and $k$.

Proof. This relies on the factorization

$$
f_{2^{k}-1}(X)=(1-X)^{k}(1+X)^{k-1} \prod_{l=1}^{k-1}\left(1+X^{2^{l}}\right)^{k-1-l} .
$$

derived from $(7-1)$.

Lemma 8.3. For $k \geqslant 2, f_{2^{k}-1}$ is strictly increasing on $\left[-1,-\frac{1}{3}[\right.$ and strictly decreasing on $[0,1]$.

Proof. The equation in the proof of the preceding lemma asserts that $f_{2^{k}-1}(x)>0$ for $x$ in ] $-1,1$. Moreover

$$
\frac{f_{2^{k}-1}^{\prime}(x)}{f_{2^{k}-1}(x)}=\sum_{l=0}^{k-1} \frac{-2^{l} x^{2^{l}-1}}{1-x^{2^{l}}}
$$

We deduce from this that $f_{2^{k}-1}^{\prime}(x)<0$ for $x \in[0,1[$ and that $f_{2^{k}-1}^{\prime}(x)>0$ on $\left[-1,-\frac{1}{3}[\right.$.

Now we prove that the derivative of Thue-Morse polynomials can be bounded, in some cases, on an neighbourhood of -1 and 1 . We set $I_{n}^{-}=[-1,-1+$ $\left.\frac{3}{2 n}\right]$ and $I_{n}^{+}=\left[1-\frac{3}{2 n}, 1\right]$.

Lemma 8.4. If $n \geqslant 3$ is an odd integer, we have $\left|f_{n}^{\prime}(x)\right| \leqslant 4$ on $I_{n}^{+}$. If $n \equiv 3 \bmod 4$ then $\left|f_{n}^{\prime}(x)\right| \leqslant 10$ on $I_{n}^{-}$.

Proof. For the first point, suppose that $n \equiv 3 \bmod 4$. Then,

$$
f_{n}(x)=f_{3}(x) g\left(x^{4}\right)=\left(1-x^{2}\right) h(x) .
$$

It is easy to see that $g(x)$ and so also

$$
h(x)=(1+x) g\left(x^{4}\right)
$$

have coefficients \pm 1 . The equality (8-1) then implies that $|h(x)| \leqslant(n+1) / 2$ on $[0,1]$ and that

$$
\left|h^{\prime}(x)\right| \leqslant \sum_{i=0}^{\frac{n-3}{4}}(4 i+4 i+1)=\frac{n^{2}-n-2}{4} .
$$

Since $f_{n}^{\prime}(x)=(1-x)^{2} h^{\prime}(x)-2(1-x) h(x)$, we have

$$
\begin{aligned}
\left|f_{n}^{\prime}(x)\right| & \leqslant\left(\frac{3}{2 n}\right)^{2} \frac{n^{2}-n-2}{4}+2 \cdot \frac{3}{2 n} \cdot \frac{n+1}{2} \\
& =\frac{33 n^{2}+15 n-18}{16 n^{2}}<3
\end{aligned}
$$

for all $n>0$.

If $n \equiv 1 \bmod 4$ we write

$$
f_{n}^{\prime}(x)=f_{n-2}^{\prime}(x) \pm\left((n-1) x^{n-2}-n x^{n-1}\right) .
$$

Obviously, for $n \geqslant 3,\left|(n-1) x^{n-2}-n x^{n-1}\right| \leqslant 1$ on $[0,1]$. The result is proved on $I_{n}^{+}$since $\left|f_{n-2}^{\prime}(x)\right|<3$, by the previous point.

For $x \in I_{n}^{-}$we begin by the case $n \equiv 7 \bmod 8$, we write $f_{n}(x)=(1+x)^{2} k(x)$ and as previously we get $|k(x)| \leqslant 2 n+2$ and $\left|k^{\prime}(x)\right| \leqslant n^{2}-n-2$ which assert that $\left|f_{n}^{\prime}(x)\right|<4$ for all $n>0$.

If $n \equiv 3 \bmod 8$, relation (7-2) ensures that

$\left|f_{n}^{\prime}(x)\right| \leqslant\left|f_{n-4}(x)\right|+\left|(n-3) x^{n-4} f_{3}(x)\right|+\left|x^{n-3} f_{3}^{\prime}(x)\right|$.

The summands are bounded by 4,2 , and 4 .

\section{THUE-MORSE POLYNOMIALS OF EVEN DEGREE}

If $n$ is a positive integer we have

$$
\begin{aligned}
& f_{2 n}(X)=f_{n}\left(X^{2}\right)-X f_{n-1}\left(X^{2}\right), \\
& f_{2 n}(X)=(1-X) f_{n}\left(X^{2}\right)+\varepsilon_{n} X^{2 n+1} .
\end{aligned}
$$

We continue with a result on the monotonicity of $f_{n}^{\prime}$ near -1 and 1 .

Lemma 9.1. Let $n$ be an even positive integer. Then $f_{n}^{\prime}$ does not vanish on $I_{n}^{-} \cup I_{n}^{+}$. More exactly, on $I_{n}^{-}$ we have

$$
\operatorname{sgn}\left(f_{n}^{\prime}\right)=\left\{\begin{aligned}
\varepsilon_{n} & \text { if } n \equiv 2 \bmod 4, \\
-\varepsilon_{n} & \text { if } n \equiv 0 \bmod 4,
\end{aligned}\right.
$$

and on $I_{n}^{+}$we have $\operatorname{sgn}\left(f_{n}^{\prime}\right)=\varepsilon_{n}$.

Proof. For $x \in I_{n}^{+}$, we write $f_{n}^{\prime}(x)=f_{n-1}^{\prime}(x)+$ $\varepsilon_{n} n x^{n-1}$. Then for $n$ large, Lemma 8.4 ensures that $n x^{n-1}$ is greater than $\left|f_{n}^{\prime}(x)\right|$ on $I_{n}^{+}$. For small $n$ we check the result directly. On $I_{n}^{-}$, the starting relations are $f_{n}^{\prime}(x)=f_{n+1}^{\prime}(x)-\varepsilon_{n+1}(n+1) x^{n}$ or 
$f_{n}^{\prime}(x)=f_{n-1}^{\prime}(x)+\varepsilon_{n} n x^{n-1}$ and we conclude with the same arguments.

Concerning the real roots of $f_{n}$, two criteria must be taken into account, namely the remainder of $n$ modulo 4 and the coefficient of the highest term $\varepsilon_{n}$ of $f_{n}$. So we define four sets: $\mathcal{A}_{0}, \mathcal{A}_{1}, \mathcal{B}_{0}$, and $\mathcal{B}_{1}$. The letter $\mathcal{A}$ stands for the condition $n \equiv 0 \bmod 4$, and $\mathcal{B}$ for $n \equiv 2 \bmod 4$. The indices 0 and 1 mean respectively that $\varepsilon_{n}=1$ and $\varepsilon_{n}=-1$. For example,

$$
\mathcal{A}_{1}=\left\{f_{n} \mid n \equiv 0 \bmod 4 \text { and } \varepsilon_{n}=-1\right\} \text {. }
$$

Figure 2 shows the behaviour of polynomials in each set.

Therefore we shall show that for $n$ even, $f_{n}$ has at most 2 real roots. The proof of this fact requires different methods depending on the part of $[-2,2]$ that we consider.

The next lemma enables us to reduce the domain where we can expect $f_{n}$ to vanish.
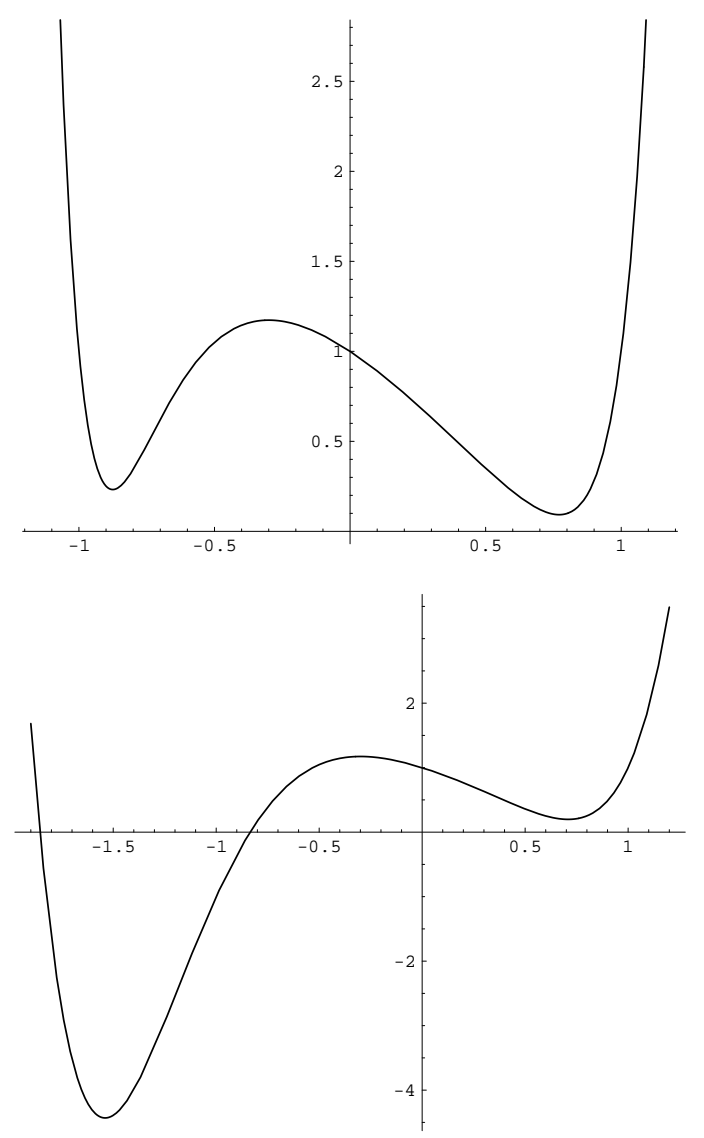

FIGURE 2. Typical representatives of $\mathcal{A}_{0}, \mathcal{A}_{1}, \mathcal{B}_{0}, \mathcal{B}_{1}$.
Lemma 9.2. Let $f_{n}$ be a Thue-Morse polynomial of even degree, then $\mathcal{R}\left(f_{n}\right) \subset[-2,1]$. In addition, if $n \equiv 0 \bmod 4$ then $\mathcal{R}\left(f_{n}\right) \subset[-1,1]$.

Proof. As $n-1$ is odd, Lemma 8.1 ensures that

$$
\left|f_{n}(x)\right| \geqslant|| x^{n}|-| f_{1}(x) f_{v}\left(x^{2}\right)|| .
$$

Now if $x>1$ then $\left|x^{n}\right|>\frac{|x-1|\left|x^{n}-1\right|}{\left|x^{2}-1\right|}$. This and (9-1) imply that $f_{n}$ has no real root greater than 1 .

If $n \equiv 0 \bmod 4$, Lemma 8.1 shows that $\left|f_{n}(x)\right| \geqslant$ ||$x^{n}|-| f_{3}(x) f_{v}\left(x^{4}\right)||$. Moreover if $|x|>1$ it is immediate that

$$
\left|x^{n}\right|>\left|\frac{(x-1)\left(x^{n}-1\right)}{x^{2}+1}\right|=\left|\frac{f_{3}(x)\left(x^{n}-1\right)}{x^{4}-1}\right| .
$$

Therefore $\left|x^{n}\right|-\left|f_{3}(x) f_{v}\left(x^{4}\right)\right|>0$ if $|x|>1$, which completes the proof.

Lemma 9.3. Let $f_{n} \in \mathcal{A}_{0} \cup \mathcal{B}_{0}$, then $f_{n}$ is positive on $[0,1]$. In particular, $\mathcal{R}\left(f_{n}\right) \cap[0,1]=\varnothing$.

Proof. We consider the set $\mathcal{C}_{0}=\mathcal{A}_{0} \cup \mathcal{B}_{0}$, ordered by the degree.
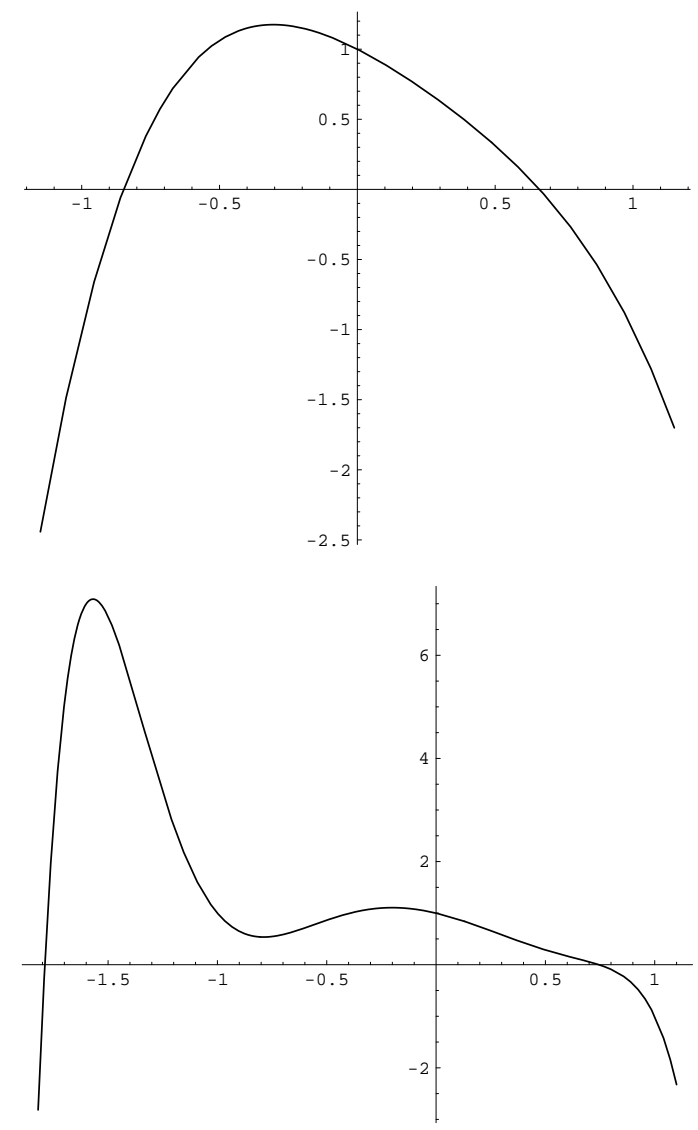
We prove the lemma by induction on the rank of the elements of $\mathcal{C}_{0}$. This result is true for the first polynomials of $\mathcal{C}_{0}$, namely, $f_{6}, f_{10}$ and $f_{12}$.

Suppose the result is true for all polynomials in $\mathrm{C}_{0}$ whose rank is at most $r \geqslant 3$. Let $d$ be the degree of the polynomial of rank $r$ and $f_{n}$ the polynomial of $\mathcal{C}_{0}$ whose rank is $r+1$. Examining $\varphi^{3}(+)$ and $\varphi^{3}(-)$ we deduce that

$$
n-d \leqslant 6 .
$$

From (9) we know that

$$
f_{n}(x)=(1-x) f_{n / 2}\left(x^{2}\right)+x^{n+1} .
$$

If $f_{n} \in \mathcal{A}_{0}$ then $f_{n / 2} \in \mathcal{C}_{0}$ and by the hypothesis of induction, $f_{n / 2}(x)>0$ on $[0,1]$. Therefore $f_{n}$ is positive on $[0,1]$, and the condition is fulfilled for a polynomial of rank $r+1$.

If $f_{n} \in \mathcal{B}_{0}$, we search the Thue-Morse polynomial of smallest degree $m$ greater than $n$ such that $f_{m} \in$ $\mathcal{A}_{0}$. The idea is to show that the difference $f_{n}(x)-$ $f_{m}(x)$ is positive. We check that $m$ must satisfy $m=$ $n+2$ or $m=n+6$ and therefore that $f_{n}(x)-f_{m}(x)$ must equal $x^{n+1}-x^{n+2}$ or

$$
x^{n+1}+x^{n+2}-x^{n+3}-x^{n+4}+x^{n+5}-x^{n+6} .
$$

Moreover, from (9-2), we have $m-d \leqslant 12$. Since $12 \leqslant d$,

$$
\frac{m}{2} \leqslant d
$$

So we can write

$$
f_{m}(x)=(1-x) f_{m / 2}\left(x^{2}\right)+\varepsilon_{m} x^{m+1}
$$

and conclude that $f_{m}$ does not vanish on $[0,1]$.

To complete the proof, we verify that $x^{n+1}-x^{n+2}$ and $x^{n+1}+x^{n+2}-x^{n+3}-x^{n+4}+x^{n+5}-x^{n+6}$ are positive on $] 0,1$. Since $f_{n}(0)=f_{n}(1)=1$, the hypothesis is fulfilled in this case too. The polynomial $f_{n}$ of rank $r+1$ does not vanish on $[0,1]$.

Lemma 9.4. Let $f_{n} \in \mathcal{A}_{0} \cup \mathcal{B}_{1}$, then $f_{n}$ is positive on $[-1,0]$. In particular, $\mathcal{R}\left(f_{n}\right) \cap[-1,0]=\varnothing$.

Proof. We consider the set

$$
\mathcal{A}_{0}^{-}=\left\{f_{n} \in \mathcal{A}_{0} \mid \varepsilon_{n-1}=-1\right\},
$$

ordered by the degree. So the terms of $f_{n}$ of degree $n$ to $n-4$ are known. They correspond to the factor ${ }_{n-4} \mathcal{E}_{n}=\psi(-)+$.

First we show by a descending induction that every polynomial in $\mathcal{A}_{0}^{-}$is positive on $\left[-1,-\frac{3}{4}\right]$.
For this, we display polynomials in $\mathcal{A}_{0}^{-}$which are positive on $\left[-1,-\frac{3}{4}\right]$ and whose degree is arbitrarily large. If $n_{k}=2^{k+2}+2^{k+1}$ with $k$ odd, then $f_{n_{k}} \in \mathcal{A}_{0}$, $\varepsilon_{n_{k}-1}=-1$ and we are able to prove that $f_{n_{k}}$ is positive on $\left[-1,-\frac{3}{4}\right]$.

Now we focus our attention on the second step of the induction. We suppose that $f_{n}$, the polynomial of rank $r$ in $\mathcal{A}_{0}^{-}$is positive on $\left[-1,-\frac{3}{4}\right]$ and we want to deduce that $f_{m} \in \mathcal{A}_{0}^{-}$whose rank is $r-1$ is also positive on $\left[-1,-\frac{3}{4}\right]$.

In fact the terms of higher degree of $f_{n}$ are well known. Examining $\mathcal{E}$ we find four cases:

$$
\begin{aligned}
& { }_{n-12} \mathcal{E}_{n}=\psi(-+-)+, \\
& { }_{n-16} \mathcal{E}_{n}=\psi(-++-)+, \\
& { }_{n-16} \mathcal{E}_{n}=\psi(-+--)+, \\
& { }_{n-20} \mathcal{E}_{n}=\psi(-++--)+.
\end{aligned}
$$

We obtain respectively $m=n-8, m=n-12$, $m=n-12, m=n-16$, so that

$$
f_{m}(x)=f_{n}(x)+x^{m+1} P(x),
$$

where $P(x)$ must be a polynomial among four determined polynomials. For example, if $m=n-8$ then $P(x)=1+x-x^{2}+x^{3}-x^{4}-x^{5}+x^{6}-x^{7}$. We point out that $m+1$ is odd and that $P(x)$ is negative on $\left[-1,-\frac{3}{4}\right]$ in all the cases. Therefore the hypothesis $f_{n}(x)>0$ proves that $f_{m}(x)>0$ on $\left[-1,-\frac{3}{4}\right]$. Every polynomial in $\mathcal{A}_{0}^{-}$is then positive on $\left[-1,-\frac{3}{4}\right]$.

If $f_{n} \in \mathcal{A}_{0} \backslash \mathcal{A}_{0}^{-}$, we see that ${ }_{n-4} \mathcal{E}_{n+11}=\psi(++-+)$ or ${ }_{n-4} \varepsilon_{n+15}=\psi(++--+)$. Then

$$
f_{n}(x)=f_{m}(x)+x^{m+1} P(x)
$$

with $f_{m}(x) \in \mathcal{A}_{0}^{-}, m=n+8$ or $m=n+12$ and $P(x)$ negative on $\left[-1,-\frac{3}{4}\right]$ in all the cases. It follows that $f_{n}(x)>0$ on $\left[-1,-\frac{3}{4}\right]$ for every polynomial $f_{n} \in \mathcal{A}_{0}$.

Finally, if $f_{n} \in \mathcal{B}_{1}$ then $f_{n-2} \in \mathcal{A}_{0}$ and $f_{n}(x)-$ $f_{n-2}(x)$ is positive on $\left.]-1,-\frac{3}{4}\right]$ which ensures that every polynomial in $\mathcal{A}_{0} \cup \mathcal{B}_{1}$ is positive on $\left[-1,-\frac{3}{4}\right]$ since $f_{n}(-1)=-1$.

Let $f_{n}$ be in $\mathcal{A}_{0} \cup \mathcal{B}_{1}$. If $n \leqslant 255$ a direct study with the command polsturm of PARI-GP shows that $f_{n}$ does not vanish on $[-1,0]$. If $n>255$, Lemma 7.1 proves that $f_{n}$ does not vanish on $\left[-\frac{3}{4}, 0\right] \subset$ $[-0.95,0]$ so that $\mathcal{R}\left(f_{n}\right) \cap[-1,0]=\varnothing$.

The two following lemmas display intervals containing a root. 
Lemma 9.5. Let $f_{n} \in \mathcal{A}_{1} \cup \mathcal{B}_{1}$, then $f_{n}$ has a unique root in $[0,1]$.

Proof. Let $\mathcal{C}_{1}=\mathcal{A}_{1} \cup \mathcal{B}_{1}$ be ordered by the degree. If $f_{n} \in \mathcal{C}_{1}$ we show that $f_{n}^{\prime}(x)<0$ on $\left[\frac{1}{2}, 1\right]$ by a descending induction on the rank of the elements of $\mathrm{e}_{1}$.

First of all, for $k \geqslant 2, f_{2^{k}}$ belongs to $\mathcal{A}_{1}$. Moreover, Lemma 8.3 asserts that $f_{2^{k}}^{\prime}<0$ on $[0,1]$. So we can start the induction at degrees arbitrarily large.

If $f_{n}$, the polynomial of rank $r$ of $\mathcal{C}_{1}$, is strictly decreasing on $\left[\frac{1}{2}, 1\right]$, then we prove that $f_{m}$, the polynomial of rank $r-1$ of $\mathcal{C}_{1}$, satisfies the same property.

If $f_{n} \in \mathcal{A}_{1}$, the tail of $f_{n}$ corresponds to the factor

$$
{ }_{n-4} \mathcal{E}_{n}=\psi(+)-\text { or }{ }_{n-4} \mathcal{E}_{n}=\psi(-)-
$$

so that $m=n-2$ or $m=n-4$.

If $f_{n} \in \mathcal{B}_{1}$, the tail of $f_{n}$ must be

$$
{ }_{n-6} \mathcal{E}_{n}=\psi(-)+-- \text { or }_{n-6} \mathcal{E}_{n}=\psi(+)+--
$$

and $m=n-6$ or $m=n-4$.

So

$$
f_{m}(x)=f_{n}(x)+x^{m+1} Q(x)
$$

with 3 possibilities for $Q(x)$. In each case, it is easy to check that $x^{m+1} Q(x)$ is decreasing on $\left[\frac{1}{2}, 1-\frac{3}{2 m}\right]$, because $Q(x)$ and $x Q^{\prime}(x)$ are increasing on this interval. On $I_{m}^{+}$, Lemma 9.1 asserts that $f_{m}^{\prime}(x)<0$.

So each polynomial of $f_{m} \in \mathcal{C}_{1}$ is strictly decreasing on $\left[\frac{1}{2}, 1\right]$. Since $f_{m}$ satisfies

$$
f_{m}(0) f_{m}(-1)=-1
$$

and does not vanish on $\left[0, \frac{1}{2}\right]$, we have proved the result.

Lemma 9.6. Let $f_{n} \in \mathcal{A}_{1} \cup \mathcal{B}_{0}$. Then $f_{n}$ has a unique root in $[-1,0]$.

Proof. For $n \leqslant 255$ a direct study shows that every $f_{n} \in \mathcal{A}_{1} \cup \mathcal{B}_{0}$ has a unique root in $[-1,0]$. We suppose until the end of the proof that $n>255$.

Let

$$
\mathcal{A}_{1}^{+}=\left\{f_{n} \in \mathcal{A}_{1} \mid \varepsilon_{n-1}=1\right\}
$$

be ordered by the degree. We show with the help of a descending induction that every element of $\mathcal{A}_{1}^{+}$ with a degree greater than 255 is strictly increasing on $[-1,-0.92]$.

First, if $k \geqslant 2$ is an even integer then $f_{2^{k}}$ belongs to $\mathcal{A}_{1}^{+}$and $f_{2^{k}}^{\prime}(x)>0$ on $[-1,-0.92]$, by Lemma 8.3 .
The second step of the induction consists in proving that $f_{m}$, the polynomial of $\mathcal{A}_{1}^{+}$of rank $r-1$, is increasing on $[-1,-0.92]$ assuming that $f_{n}$ the polynomial of rank $r$ is also increasing on this interval. The different possible tails of $f_{n}$ are

$$
\begin{aligned}
& { }_{n-12} \mathcal{E}_{n}=\psi(+-+)-, \\
& { }_{n-16} \mathcal{E}_{n}=\psi(+--+)-, \\
& { }_{n-16} \mathcal{E}_{n}=\psi(+-++)-, \\
& { }_{n-20} \mathcal{E}_{n}=\psi(+--++)-,
\end{aligned}
$$

so that

$$
f_{m}(x)=f_{n}(x)+x^{m+1} R(x),
$$

where $R(x)$ can take four different values. In each case, we check that $x^{m+1} R(x)$ is strictly increasing on $[-1+3 / 2 m,-0.92]$.

As Lemma 9.1 proves that $f_{m}^{\prime}(x)>0$ on $I_{m}^{-}$, the monotonicity of $f_{m}(x)$ on $[-1,-0.92]$ is established for all the polynomials in $\mathcal{A}_{1}^{+}$of degree $>255$.

If $f_{n} \in \mathcal{A}_{1} \backslash \mathcal{A}_{1}^{+}$we point out that ${ }_{n-4} \varepsilon_{n+11}=$ $\psi(--+-)$ or ${ }_{n-4} \mathcal{E}_{n+15}=\psi(--++-)$. So

$$
f_{n}(x)=f_{m}(x)+x^{m+1} R(x)
$$

with $f_{m}(x) \in \mathcal{A}_{1}^{+}, m=n+8$ or $m=n+12$ and $x^{m+1} R(x)$ strictly increasing on $[-1+3 / 2 m,-0.92]$. Thus as previously, $f_{n}^{\prime}(x)>0$ on $[-1,-0.92]$.

Finally, $f_{n} \in \mathcal{A}_{1}$ is equivalent to $f_{n+2} \in \mathcal{B}_{0}$. Now $f_{n+2}(x)-f_{n}(x)$ is increasing on

$$
[-1+3 /(2 n+4),-0.92],
$$

and we conclude on $I_{n+2}^{-}$by Lemma 9.1.

So every polynomial $f_{n} \in \mathcal{A}_{1} \cup \mathcal{B}_{0}$ is strictly increasing on $[-1,-0.92]$. From Lemma $7.1, f_{n}$ does not vanish on $[-0.92,0]$ and it satisfies

$$
f_{n}(0) f_{n}(-1)=-1 \text {. }
$$

So $f_{n}$ has a unique root in $[-1,0]$.

We now study the property of the reciprocal polynomial $f_{n}^{*}$ of $f_{n}$ in order to determine the real roots of $f_{n}$ outside the unit circle.

Lemma 9.7. Let $f_{n} \in \mathcal{B}_{0} \cup \mathcal{B}_{1}$. We can then write

$$
f_{n}(X)=\varepsilon_{n} Q(X) X^{n-\operatorname{deg} Q}+f_{r}(X),
$$

with $r=n-\operatorname{deg} Q-1$ and where $Q(X)$ is one of $f_{10}(X),-f_{14}(X), f_{18}(X),-f_{22}(X)$.

Proof. For convenience, we first prove a similar result for $m \equiv 3 \bmod 4$. 
If $m \equiv 3 \bmod 4$ then $f_{m}(X)=f_{3}(X) f_{v}\left(X^{4}\right)$. Without loss of generality we can assume that $\varepsilon_{m}=$ 1. As $\psi(+-+-+)$ never occurs in the Thue-Morse word $\mathcal{E}$, we see that the tail of $f_{m}$ must be one of the following words

$$
\begin{aligned}
& { }_{m-11} \mathcal{E}_{m}=\psi(-++), \\
& { }_{m-15} \mathcal{E}_{m}=\psi(+--+), \\
& { }_{m-19} \mathcal{E}_{m}=\psi(-++-+), \\
& { }_{m-23} \mathcal{E}_{m}=\psi(+--+-+) .
\end{aligned}
$$

Thus $f_{m}(X)$ equals

$$
P(X) X^{m-\operatorname{deg} P}+f_{r}(X),
$$

where $P(X)$ is one of $-f_{11}(X), f_{14}(X),-f_{18}(X)$, or $f_{22}(X)$. If $n=m-1$ then

$$
f_{n}(X)=Q(X) X^{n-\operatorname{deg} Q}+f_{r}(X),
$$

where $Q(X)$ is one of $-f_{10}(X), f_{14}(X),-f_{18}(X)$, or $f_{22}(X)$. Finally, $\varepsilon_{m}=-\varepsilon_{n}$ and the proof is completed.

Lemma 9.8. Let $f_{n} \in \mathcal{B}_{0} \cup \mathcal{B}_{1}$. Then

$$
\mathcal{R}\left(f_{n}\right) \cap[-1.3,-1]=\varnothing .
$$

Proof. Let $f_{n} \in \mathcal{B}_{0} \cup \mathcal{B}_{1}$. Using Lemma 9.7 we write

$$
f_{n}(x)=\varepsilon_{n} Q(x) x^{n-\operatorname{deg} Q}+f_{r}(x),
$$

with $Q \in\left\{-f_{10}, f_{14},-f_{18}, f_{22}\right\}$ and

$$
r=n-\operatorname{deg} Q-1 \equiv 3 \bmod 4 .
$$

If $f_{n}$ vanishes at $x$ then $x^{n-\operatorname{deg} Q}|Q(x)|=\left|f_{r}(x)\right|$ and

$$
\begin{aligned}
\left|f_{r}(x)\right| & \leqslant\left|\frac{\left(x^{2}-1\right)\left(x^{n-\operatorname{deg} Q}\right)}{x^{2}+1}\right| \leqslant\left|x^{n-\operatorname{deg} Q}-1\right| \\
& =x^{n-\operatorname{deg} Q}-1 .
\end{aligned}
$$

Thus

$$
|Q(x)| \leqslant \frac{x^{n-\operatorname{deg} Q}-1}{x^{n-\operatorname{deg} Q}} \leqslant 1 .
$$

Therefore if $|Q(x)|>1$ then $f_{n}(x) \neq 0$. Now the minimum of

$$
-f_{10}(x), f_{14}(x),-f_{18}(x), f_{22}(x)
$$

is $>1$ on $\left[-1.3,-1\left[\right.\right.$. Since $f_{n}(-1) \neq 0$ we have $\mathcal{R}\left(f_{n}\right) \cap[-1.3,-1]=\varnothing$.

Lemma 9.9. If $f_{n} \in \mathcal{B}_{0} \cup \mathcal{B}_{1}$ then $f_{n}$ has a unique root in $[-2,-1]$.
Proof. By Lemma 9.7,

$$
f_{n}^{*}(x)-\varepsilon_{n} Q^{*}(x)=f_{r}^{*}(x) x^{\operatorname{deg} Q+1}
$$

with $r=n-\operatorname{deg} Q-1 \equiv 3 \bmod 4$. Therefore

$$
f_{r}^{*}(x)=f_{3}(x) g\left(x^{4}\right),
$$

where $g$ is a unimodular polynomial. Thus

$$
\left|\left(f_{r}^{*}(x) x^{\operatorname{deg} Q+1}\right)^{\prime}\right| \leqslant \sum_{k=0}^{v}\left|\left(x^{4 k} f_{3}(x) x^{\operatorname{deg} Q+1}\right)^{\prime}\right| .
$$

Now for $n$ odd and $x$ in $[-0.8,-0.5],\left|\left(f_{3}(x) x^{n}\right)^{\prime}\right| \leqslant$ $2.52(-0.8)^{n}-0.81(-0.8)^{n} n$. As

$\sum_{k=0}^{\infty} 2.52(-0.8)^{4 k+11}-0.81(-0.8)^{4 k+11}(4 k+11)<1.3$,

we deduce that on this interval $\left|\left(f_{r}^{*}(x) x^{\operatorname{deg} Q+1}\right)^{\prime}\right|<$ 1.3 as soon as $\operatorname{deg} Q \geqslant 10$. The study of the derivatives of $f_{10}^{*},-f_{14}^{*}, f_{18}^{*}$ and $-f_{22}^{*}$, ensures that they are greater than $1.3671 \ldots$ in modulus and that they keep the same sign on $[-0.8,-0.5]$. The derivative of $f_{n}^{*}$ then keeps its sign on $[-0.8,-0.5]$. Now

$$
f_{n}^{*}(0) f_{n}^{*}(-1)=-1
$$

and $f_{n}^{*}(x)$ does not vanish on $[-1,-0.8]$ by Lemma 9.8 . Thus $f_{n}^{*}$ has a unique real root in $[-1,-0.5]$. This proves that $f_{n}$ vanishes only once in $[-2,-1]$.

We can now prove Theorems 6.1 and 6.2.

\section{PROOF OF THE THEOREMS}

Proof of Theorem 6.1. Let $n$ be even. Lemmas 9.2, 9.3, 9.4 establish the theorem when $f_{n} \in \mathcal{A}_{0}$.

If $f_{n} \in \mathcal{A}_{1}$, Lemmas $9.2,9.5$ and 9.6 ensure the result.

If $f_{n} \in \mathcal{B}_{0}$, Lemmas 9.2, 9.3, 9.6 and 9.9 allow us to conclude.

If $f_{n} \in \mathcal{B}_{1}$, Lemmas $9.2,9.4,9.5$ and 9.9 complete the proof for the case $n$ even.

Let $n$ be odd. If $k$ is the 2 -adic valuation of $n+1$ then we see that

$$
f_{n}(X)=f_{2^{k}-1}(X) f_{v}\left(X^{2^{k}}\right)
$$

with $v$ even. From what we have just proved $f_{v}$ has a real positive root if and only if $\varepsilon_{v}=-1$. The relation $\varepsilon_{2^{k}-1}=(-1)^{k}$ and Lemma 8.2 complete the proof. 
Proof of Theorem 6.2. Let $N$ be an integer. We investigate polynomials of even degree $n<N$. The relation $\left|f_{N-1}(1)\right| \leqslant 1$ implies that

$$
\frac{N-1}{2} \leqslant\left|\left\{0 \leqslant i \leqslant N-1 \mid \varepsilon_{i}=1\right\}\right| \leqslant \frac{N+1}{2} .
$$

The same inequalities hold for

$$
\left|\left\{0 \leqslant i \leqslant N-1 \mid \varepsilon_{i}=-1\right\}\right| .
$$

Since $\varepsilon_{i}=1$ is equivalent to $\varepsilon_{2 i}=1$ and $\varepsilon_{i}=1$ is equivalent to $\varepsilon_{2 i+1}=-1$, the four sets

$$
\left\{0 \leqslant i \leqslant N-1 \mid \varepsilon_{i}=(-1)^{r} \text { and } i \equiv s \bmod 4\right\},
$$

for $r \in\{0,1\}$ and $s \in\{0,2\}$, have between $N / 8-C$ and $N / 8+C$ elements, for some constant $C$ independent of $N$. So the Thue-Morse polynomials of even degree less than $N$ contribute to at least $\frac{3 N}{4}-6 C$ and to at most $\frac{3 N}{4}+6 C$ real roots.

We now examine odd degrees. Let $k$ be an integer. We consider the integers $i \in[0, N-1]$ such that the 2 -adic valuation of $i+1$ is precisely $k$. These $i$ 's can be written generically $q 2^{k+1}+2^{k-1}$ with $q \in \mathbb{N}$ and $\varepsilon_{i}=\varepsilon_{q}(-1)^{k}$. So for $q \equiv 0 \bmod 4$ and $t=q, q+1, q+2, q+3$, the Thue-Morse polynomials of degree $t 2^{k+1}+2^{k-1}$ have $8 k$ roots. Now for every $N$ and $k$ fixed, one can make $\left\lfloor N / 2^{k+3}\right\rfloor$ such groups which give $8 k\left\lfloor N / 2^{k+3}\right\rfloor$ roots, and for each $k$ we forget at most $8 k$ roots in the sum.

Thus it is easy to see that

$$
\begin{aligned}
-\frac{6 C}{N} & \leqslant \frac{1}{N} \sum_{n=0}^{N-1} \rho\left(f_{n}\right)-\frac{1}{N}\left(\sum_{k=0}^{\infty} 8 k\left\lfloor\frac{N}{2^{k+3}}\right\rfloor+\frac{3 N}{4}\right) \\
& \leqslant \frac{6 C}{N}+\frac{1}{N} \sum_{k=0}^{\left\lfloor\log _{2} N\right\rfloor} 8 k
\end{aligned}
$$

which ensures that

$$
\lim _{N \rightarrow \infty} \frac{1}{N} \sum_{n=0}^{N-1} \rho\left(f_{n}\right)=\frac{11}{4} .
$$

\section{ACKNOWLEDGEMENTS}

We were greatly helped in this work by Laurent Habsieger. Camille Laurent-Gengoux $[\geq 2000]$ and Jian-yan Yao extended the results of [Mendès France 1991] to higher dimensions. We thank them wholeheartedly.

\section{ADDENDUM}

After this article was submitted, a generalization of its methods allowed Doche to find families of $(+,-)$ sequences for which

$$
\liminf _{n \rightarrow \infty} \rho\left(f_{n}\right) / \log n>0 .
$$

See [Doche 1999] for details.

\section{REFERENCES}

[Dekking and Mendès France 1981] F. M. Dekking and M. Mendès France, "Uniform distribution modulo one: a geometrical viewpoint", J. Reine Angew. Math. 329 (1981), 143-153.

[Doche 1999] C. Doche, "On the real roots of generalized Thue-Morse polynomials", preprint, 1999. Submitted for publication.

[Edelman and Kostlan 1995] A. Edelman and E. Kostlan, "How many zeros of a random polynomial are real?", Bull. Amer. Math. Soc. (N.S.) 32:1 (1995), 1-37. Erratum in 33:3 (1996), 325.

[Erdős and Offord 1956] P. Erdős and A. C. Offord, "On the number of real roots of a random algebraic equation", Proc. London Math. Soc. (3) 6 (1956), 139160.

[Favard 1932] J. Favard, "Une définition de la longueur et de l'aire", C. R. Acad. Sci. Paris 194 (1932), 344346.

[Kac 1943] M. Kac, "On the average number of real roots of a random algebraic equation", Bull. Amer. Math. Soc. 49 (1943), 314-320. Correction in 49 (1943), 938.

[Kac 1949] M. Kac, "On the average number of real roots of a random algebraic equation, II", Proc. London Math. Soc. (2) 50 (1949), 390-408.

[Kac 1959] M. Kac, Probability and related topics in physical sciences, Lectures in Appl. Math. 1A, Interscience, New York, 1959. Reprinted by the Amer. Math. Soc., 1976.

[Klain and Rota 1997] D. A. Klain and G.-C. Rota, Introduction to geometric probability, Lezione Lincee, Cambridge University Press, Cambridge, 1997.

[Laurent-Gengoux $\geq 2000]$ C. Laurent-Gengoux, "Courbes du plan et droites aléatoires", Jour. math. des élèves ENS. Submitted.

[Mendès France 1991] M. Mendès France, "The Planck constant of a curve", pp. 325-366 in Fractal geometry and analysis (Montreal, 1989), edited by J. Bélair and 
S. Dubuc, NATO Adv. Sci. Inst. Ser. C Math. Phys. Sci. 346, Kluwer Acad. Publ., Dordrecht, 1991.

[Mendès France and Tenenbaum 1981] M. Mendès France and G. Tenenbaum, "Dimension des courbes planes, papiers pliés et suites de Rudin-Shapiro", Bull. Soc. Math. France 109:2 (1981), 207-215.
[Santaló 1976] L. A. Santaló, Integral geometry and geometric probability, Encycl. Math. and its Appl. 1, Addison-Wesley, Reading, MA, 1976.

[Sulanke 1966] R. Sulanke, "Integralgeometrie ebener Kurvennetze", Acta Math. Acad. Sci. Hungar. 17 (1966), 233-261.

Christophe Doche, Laboratoire d'Algorithmique Arithmétique, Université Bordeaux I, 351, cours de la Libération, F-33405 Talence Cedex France (cdoche@math.u-bordeaux.fr)

Michel Mendès France, Laboratoire d'Algorithmique Arithmétique, Université Bordeaux I, 351, cours de la Libération, F-33405 Talence Cedex France (mmf@math.u-bordeaux.fr)

Received April 7, 1999; accepted in revised form October 11, 1999 